\title{
Use of Maldi-Tof Mass spectrometry in direct microorganism identification in clinical laboratories
}

\author{
Tamara Brunelli, Roberto Degl'Innocenti, Antonella Conti, Patrizia Casprini \\ Laboratorio Analisi Chimico Cliniche, Ospedale Misericordia e Dolce, ASL4 Prato
}

Key words: MALDI-TOF, spectrometry, identification, microorganism, bacteria

Uso della spettrometria di massa Maldi-Tof nell'identificazione diretta dei microrganismi nel laboratorio clinico

\section{SUMMARY}

Mass Spectrometry is an old technique that has recently been introduced in the clinical microbiology laboratory as Matrix Assisted Laser Desorption lonization Time of Flight Mass Spectrometry (MALDI-TOF MS).

MALDI is a soft ionization technique used in mass spectrometry that allows the analysis of biomolecules and large organic molecules which tend to be fragile and fragment when ionized. To obtain ions biological specimens are mixed with a matrix which specifically absorbs the ionization source (a laser beam). The high energy impact is followed by the formation of ions which are extract through an elastic field, focussed and detected as mass/charge $(\mathrm{m} / \mathrm{z})$ spectrum. The differences between ions are seen with TOF, a revelation system that relates the time of flight of a ion to the charge/mass value: ion with a higher $\mathrm{m} / \mathrm{z}$ have are slower (a bigger time of flight) than ions with lower $\mathrm{m} / \mathrm{z}$. MALDI-TOF MS, in clinical microbiology laboratory, is used to identify bacteria and fungi directly from samples.

The identification of microorganisms can be performed directly from body fluids (e.g. urine, blood culture, after centrifugation and recovery of microorganisms) or from colonies (after cultivation).

The rapidity of identification is of great importance in blood cultures. Positive cultures with one microorganism are processed in a different way than those with more than one microorganism. In positive monomicrobial cultures, after separation of microbs from blood cells, we can perform an immediate identification with MALDI-TOF MS that we can communicate to the clinician, and that gives indication to perform the correct antibiogram.

Major problems are present when more than one microorganism are in the culture: in this case we have to use the method of subcultivation and then the identification with mass-spectrometry can be performed.

MALDI-TOF MS is a rapid, reliable and low cost technique, that can identify a growing number of microorganisms. This technique can significantly reduce the time to give an identification, that is of great importance, particularly in blood culture, where the success of therapy is strictly linked to its early beginning.

\section{INTRODUCTION}

Until recently Matrix Assisted Laser Desorption Ionization Time of Flight Mass Spectrometry (MALDI-TOF MS) main research areas are the identification of proteins in the field of proteomics and the detection of biomarkers of several diseases including cancer, Alzheimer disease and allergy. In microbiology MALDI-TOF MS has been used to distinguish strains within a single species or to differentiate closely related bacteria. Even though the first study of the use of MALDITOF MS for the identification of bacteria dates back to 1985 (1), MALDI-TOF MS devices designed for use under routine conditions have only recently been commercially introduced. Due to these new devices the application of MALDI-TOF for rapid identification of Gram positive and Gram-negative microorganisms taken directly from culture has enormously increased (5). Until now in the clinical diagnostic microbiology laboratory, the identification of bacterial and yeast isolates is based on phenotypic characteristics such as growth on different media, colony morphology, Gram stain and various biochemical reactions. Altogether these techniques allow the identification of most bacterial isolates with great accuracy, but they are cost and time consuming. For its characteristics of rapidity, use of few regents and low cost MALDITOF MS is a promising technique to reduce costs and time for the identification of microorganisms.

\section{MALDI-TOF MS}

MALDI is a soft ionization technique used in mass spectrometry that allows the analysis of biomolecules (such as proteins, peptides and sugars) and large organic molecules which tend to be fragile and fragment when ionized (Figure I).

A pulsed laser (normally a nitrogen laser) is used to vaporize and ionize the sample. A matrix is used to protect the biomolecule from being destroyed by direct laser beam and to facilitate vaporisation and ionization. The laser pulse repetition rate is low (from $10 \mathrm{~Hz}$ to $1 \mathrm{kHz}$ ) but the number of ions reaching the detection is very large.

Biological specimens are mixed with a matrix (the most used is HCCA) which specifically absorbs a laser beam.

The high energy impact is followed by the formation of ions which are extract through an elastic field, focussed and detected as mass/charge $(\mathrm{m} / \mathrm{z})$ spectrum.

The laser is fired at the crystals in the MALDI spot.

The matrix absorbs the laser energy and becomes ionized, then the matrix itself transfers part off its charge to the analyte molecules (e.g. proteins), thus ionizing them while still

\section{Corresponding author: Tamara Brunelli}

Laboratorio Analisi Chimico Cliniche, Ospedale Misericordia e Dolce, ASL4 Prato

Piazza dell'Ospedale 5, 59100 Prato - Tel.: 0574434720 - Fax: 0574434774

E-mail: tbrunelli@usI4.toscana.it

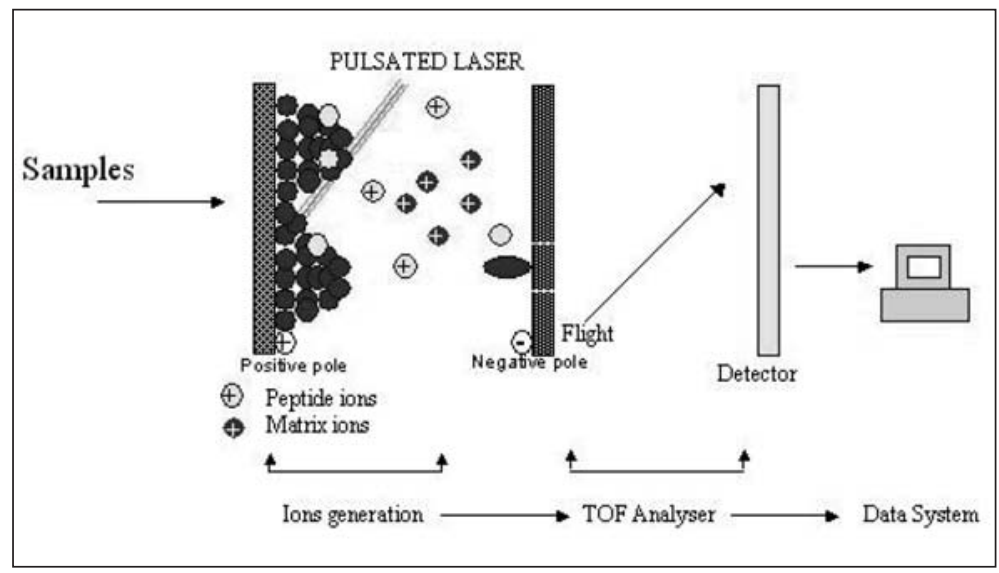

Figura I. Maldi lonization System. 
protecting them from the disruptive energy of the laser.

The type of mass spectrometer most widely used with MALDI is the TOF (time-of-flight mass spectrometer).

TOF is a revelation system that relates the time of flight of a ion to the charge/mass value: ion with a higher $\mathrm{m} / \mathrm{z}$ have are slower (a bigger time of flight) than ions with lower $\mathrm{m} / \mathrm{z}$.

MALDI-TOF instruments are usually equipped with an ion mirror, deflecting ions with an electric field, thereby doubling the ion flight path and increasing the resolution.

After separation, ions go to the detector and the data are collected in an electronic sheet.

The procedure generates protein fingerprint signatures from whole bacterial cells called "peptide spectra"; a "unique spectrum" corresponds to a microorganism ("fingerprint spectrum"). The identification of microorganisms is obtained by comparing, using algorithms, the obtained spectrum to a database containing spectra that are generated based on measurements on well known microoorganisms.

These spectra contain characteristic signals of genus, species and strain biomarkers which are representative for the respective group of microorganisms.

At present MALDI-TO MS allows the identification of most bacteria and an increasing number of fungi.

For routine bacterial isolates, a correct identification by MALDI-TOF MS at the species level was obtained in $79.9 \%$ to $93.6 \%$ of cases (2). Commercially available MALDI-TOF MS devices designed for clinical microbiology laboratories are of a size of classical laboratory device and do not require specific equipment other than a computer. They can also connect to the laboratory information system or to the other automated devices (such as the spotter that automatically spots the bacteria containing solution on the sample tray).

Two MALDI-TOF MS system are commercially available: one is from Bruker Daltonics and the other is from Shimadzu Corporation. The comparison between the two instruments has shown little differences in microorganism identification (from colonies); in fact the Bruker system gave high confidence identification for 680 isolates of 720 (of which 674, 99.1\%, were correct) and the Shimadzu system gave high confidence identification for 639 isolates (of which 635, 99.4\%, were correct) (4).

\section{DIRECT IDENTIFICATION OF MICROORGANISMS}

The identification of microorganisms can be performed directly from body fluids (e.g. urine, blood culture, after centrifugation and recovery of microorganisms) or from colonies (after cultivation) (3). Data from literature on body fluids are mainly obtained in blood cultures, but also the identification directly from urine is of interest.

As far as blood cultures are concerned, a number of papers has been published $(7,10,11,15)$. This interest is because the turnaroundtime for this "exam" is crucial: in fact the sooner the appropriate therapy begins the better are the outcomes.

Pathogen growth in blood culture vessels is continously monitored by semiautomated incubators. At present, on cultures recognized as positive, Gram's stain allows for preliminary identification.

Positive cultures with one microorganism are processed in a different way than those with more than one microorganism. In positive monomicrobial cultures, after separation of microbs from blood cells, we can perform an antiobiogram susceptibility test.

This procedure permits to obtain an immediate identification that we can communicate to the clinician, and gives indication to perform the correct antibiogram.

Major problems are present when more than one microorganism are in the culture: in this case we have to use the method of subcultivation and then the identification with mass-spectrometry can be performed.

Also in this case, this method is less time-consuming than the classical one, because as soon as the colonies are grown we can have identification without waiting for the time that we need to perform it with the classical method (that means that 24 postpositivisation of a culture with more than one microb (Figure II). The time between positivisation and the final result (identification + antibiogram) is, in both cases, significantly reduced: with MALDI-TOF it arrives in 24/48 hours, without MALDI-TOF we need 48/72 hours.

This approach using MALDI is very useful in terms of reducing the time to have a result and consequently in terms of reducing the time between positivisation of blood culture and beginning of the appropriate therapy.

When fungi are present in blood cultures direct MALDI-OF MS analysis of aliquots from positive cultures allows rapid and accurate identification of the main Candida species, thus

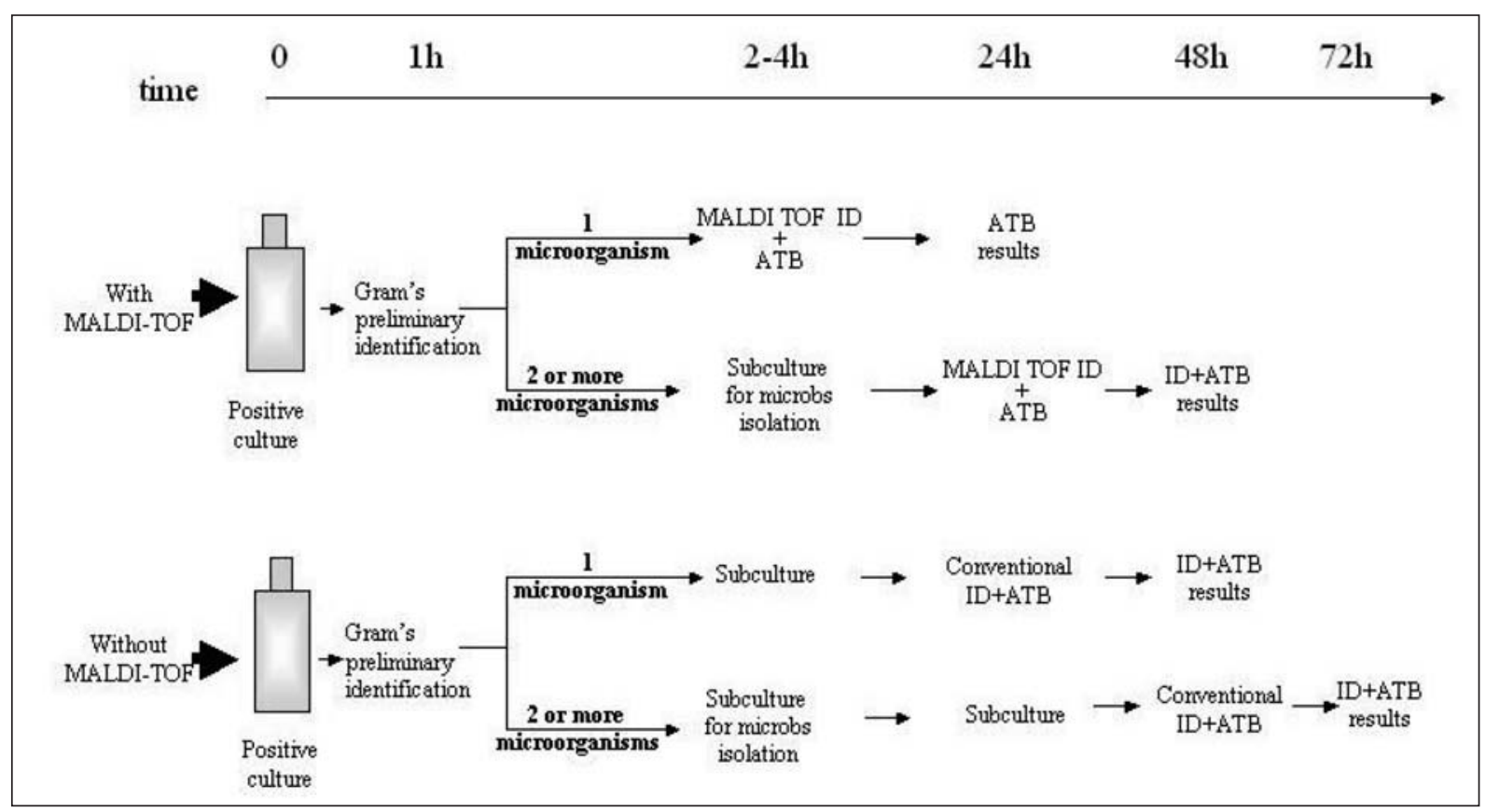

Figure II. Time course comparison for the characterisation of microorganisms in a positive blood culture with and without MALDI-TOF MS. $A T B=$ antibiogram, $I D=$ identification 
obviating the need for subculturing on specific media (14). Data from literature affirm that successful ID was performed in $66 \%$ of 562 (11), $79 \%$ of 21 (15), 76.4\% of 212 (16), 91\% of 434 (10) 95\% of 277 (6) of monomicrobial positive blood cultures.

The daily increase of the number of available spectra allows a fast growing percentage of correct identification.

In the urine sample, bacterial identification directly from infected urine is possible (9). Sample preparation is easy: we need to centrifugate two times the sample (first to remove leucocytes, second to collect bacteria). In the direct method, a small amount of bacteria is applied to the MALDI plate in a thin film.

When the direct method do not yield reliable identification, an ethanol/formic acid extraction procedure was performed. In both cases HCCA matrix was used for the detection.

The use of MALDI-TOF MS in urine samples seems to be of less importance since an important number of urine samples can be processed by quantitative automated plating on chromogenic media.

\section{CONCLUSIONS}

The need of a rapid identification of microorganisms, especially in positive blood cultures, can be satisfied using MALDI-TOF MS, a method that uses low cost reagents and need a short and easy sample preparation.

The technique is of great interest an many groups are using it; consequently the number of available MALDI-TOF MS bacteria spectra (database spectra) increases rapidly and the number of misidentification is lowering. Good results are also obtained in identification of clinically important yeast species $(14,17)$.

In addition, also the list of available devices for the automation of MALDI-TOF MS is increasing: these tools are very useful for reducing the working time of the operator and the number of possible errors due to sample handling.

MALDI-TOF MS is also promising to distinguish methicillin susceptible (MSSA) from methicillin resistant (MRSA) $S$. aureus (8) and to predict antibiotic susceptibility in bacteria (12) and yeasts (13).

Due to its characteristics, MALDI-TOF MS seems to be suitable for high-throughput and rapid diagnostics al low cost and can be considered an alternative for conventional biochemical and molecular identification systems in a routine microbiological laboratory.

\section{REFERENCES}

1. Anhalt JP, Fenselau C. Identification of bacteria using mass spectrometry. Anal Chem 1975; 47: 12-225.

2. Bizzini A, Durussel C, Bille J, Greub G, Prod'hom G. Performance of matrix-assisted laser desorption ionization-time of flight mass spectrometry for identification of bacterial strains routinely isolated in a clinical microbiology laboratory. J Clin Microbiol 2010; 48: 1549-54.

3. Bizzini A, Greub G. MALDI-TOF MS, a revolution in clinical microbial identification. Clin Microbiol Infect 2010; Jul 15 (Epub ahead of print).

4. Cherkaoui A, Hibbs J, Emonet S, et al. Comparison of two matrix-assisted laser desorption ionization-time of flight mass spectrometry methods with conventional phenotypic identification for routine identification of bacteria to the species level. J Clin Microbiol 2010; 48 (4): 1169-75.

5. Claydon MA, Davey SN, Edwards-Jones V, Gordon DB. The rapid identification of intact microorganisms using mass spectrometry. Na Biotechnol 1996; 14: 1584-6.

6. Christner M, Rohde $\mathrm{H}$, Wolters $\mathrm{M}$, et al. Rapid identification of bacteria from positive blood culture bottles by use of matrix-assisted laser desorption-ionization time of flight mass spectrometry fingerprinting. $J$ Clin Microbiol 2010; 48 (5): 1584-91.

7. Drancourt M. Detection of microorganisms in blood specimens using MALDI-TOF mass spectrometry: a review. Clin Microbiol Infect 2010; Jun 8: (Epub ahead of print).

8. Edwards-Jones V, Claydon MA, Evason DJ, et al. Rapid discrimination between methicillin-sensitive and methicillin-resistant Staphylococcus aureus by intact cell mass spectrometry. J Med Microbiol 2000; 49: 295-300.

9. Ferreira L, Sánchez-Juanes F, a González-Ávila MN, et al. Direct identification of urinary tract pathogens FROM urine samples by MALDITOF (Matrix-Assisted Laser Desorption Ionization Time-of-Flight) mass spectrometry. Clin Microbiol 2010; 48: 2110-5.

10. Ferroni A, Suarez S, Beretti JL, et al. Real time identification of bacteria and yeast in positive blood culture broths by MALDI-TOF-Mass Spectrometry. J Clin Microbiol 2010; 48: 1542-8.

11. La Scola B, Raoult D. Direct identification of bacteria in positive blood culture bottles by matrix-assisted laser desorption ionisation time-of-flight mass spectrometry. PLoS One, 2009; 4 (11): e8041.

12. Majcherczyk PA, McKenna T, Moreillon P, Vaudaux P. The discriminatory power of MALDI-TOF mass spectrometry to differentiate between isogenic teicoplanin-susceptible and teicoplanin-resistant strains of methicillinresistant Staphylococcus aureus. FEMS Microbiol Lett 2006; 255: 233-9.

13. Marinach C, Alanio A, Palous M, et al. MALDI-TOF MS-based drug susceptibility testing of pathogens: the example of Candida albicans and fluconazole. Proteomics 2009; 20: 4627-31.

14. Marinach-Patrice C, Fekkar A, Atanasova R, et al. Rapid species diagnosis for invasive candidiasis using mass spectrometry. PLoS One. 2010; 5 (1): e8862.

15. Prod'hom G, Bizzini A, Durussel C, Bille J, Greub G. Matrix-assisted lase desorption ionization-time of flight mass spectrometry for direct bacterial identification from positive blood culture pellets. J Clin Microbiol 2010; 48 (4): $1481-4$.

16. Stevenson LG, Drake SK, Murray PR. Rapid identification of bacteria in positive blood culture broths by matrix-assisted laser desorption ionizationtime of flight mass spectrometry. J Clin Microbiol 2010; 48 (2): 444-7.

17. Stevenson LG, Drake SK, Shea YR, Zelazny AM, Murray PR. Evaluation of Matrix-Assisted Laser Desorption/Ionization Time-of-Flight Mass Spectrometry (MALDI-TOF) for the Identification of Clinically Important Yeast Species. J Clin Microbiol 2010 Jul 28: [Epub ahead of print]. 